\title{
Engineering Education for the 21st century: Technology based hierarchical online learning
}

\author{
Avik W. Ghosh \\ Charles L Brown School of Electrical and Computer \\ Engineering University of Virginia, Charlottesville, VA 22904, \\ USA ag7rq@virginia.edu
}

\begin{abstract}
Engineering education in the 21st century faces a host of challenges. A new generation of students is finding itself perpetually wired, as brick and mortar buildings are competing with e-books, youtube and wikipedia, classrooms with gaming and Second Life, along with an overall push to decentralize education through Massive Open Online Courses (MOOCs) and virtual universities. We face a huge challenge and therein a unique opportunity to design platforms that can actively engage future engineers, teach useful skills and disseminate information for long-termed retention. With the proliferation of for-profit online universities like Univ. of Phoenix, and online courses by EdX, Coursera, Udacity, it is often hard to separate hope from hype when it comes to MOOCs and online learning. This paper focuses on my experiments with asynchronous and blended learning over years of engineering course design.
\end{abstract}

Keywords: MOOCs, online education, engineering design

\section{Avik W. Ghosh}

Charles L Brown School of Electrical and Computer

Engineering University of Virginia, Charlottesville, VA 22904,

USA ag7rq@virginia.edu

\section{The online platform: Separating Hope from} Hype

\section{A. Online Learning - simply a better 'textbook'?}

There is a definite plus to the online learning component of a MOOC, seen from the clear popularity of Khan Academy. At its minimum, it offers an enhanced experience with a substantially wider reach, allowing asynchronous, paced-out learning - whether 'flipped' or 'blended'. We are well past the days when we spend inordinate amounts of time in dusty libraries looking for a particular book or journal with moth-eaten pages. A large volume of material is now available readily, archived through various Big Data Initiatives. One needs only to look at Chemical Abstracts Services, MRQE (Movie Review Query Engine), Animal Genome Size database, or even-closer to home - the complete works of Rabindranath Tagore (http://bichitra.jdvu.ac.in/ index .php) to get a sense for their expansive scope. With enormous amount of effort going into data mining, visualizing and archiving, and the ease of creating and uploading information through Software as a Service (SaaS) websites like Knack, online information access and learning is here to stay. Our own efforts at database creation relates to Materials Genome - a White House Initiative, where we use high throughput computational tools such as Density Functional Theory to rapidly create and then subsequently sift through large volumes of data - bandstructures, magnetizations and thermal stabilities of almost a thousand alloys of the Heusler family. We then data 
mine for interesting properties -half-metallicity, semiconducting behavior, and compatible lattice constants for heterostructure creation. These data are currently being uploaded at databases at University of Alabama and will hook up with international databases down the road.

\section{B. From Online Learning to Online Courses - the Challenge with MOOCs}

The goal of MOOCs goes well beyond online dissemination of knowledge. The over-arching purpose, ostensibly, is the democratization and costcutting of education. This is certainly a noble cause, especially for children in third world countries for whom affordable education is the main ticket out of poverty. The potential impact of MOOCs extends to wealthier countries as well -such as the US where student debt has now ballooned upto $\$ 1$ trillion. The average cost for an in-state public college in 20142015 is $\$ 23,410$ while that of a private college is $\$ 46,272$. These numbers constitute a substantial fraction of the US median household income in 2015 at $\$ 55,794$ (data reported by Advisor Perspectives). Potentially, a MOOC could cut the cost of education substantially by the sheer volume of scale. The numbers speak for themselves - Coursera has drawn upwards of 5 million students, and edX more than 1.3 million. The large scale dissemination of coursework is expected to significantly reduce the administrative overheads associated with conventional courseware. There is thus a clear expectation of rapid, disruptive changes happening in the world of education, the same way the music industry was up-ended almost overnight with P2P file sharing through Napster.

And yet, the cheap commodification of knowledge has had a rocky start, to say the least. So far, there is no evidence that conventional education is on its way out. The much-discussed partnership between San Jose State and Udacity targeting high-risk students ended up with substantially lower (24-51\%) pass rates compared to historical in-person rates (46-76\%). A popular account that proponents of MOOCs like to cite is that courses in certain highly ranked universities tally over 100,000 student sign-ups. However, despite the courses being free (or perhaps because of it), $\sim 95 \%$ students did not complete the courses. A few of the challenges and opportunities of MOOCs are listed below: o Incentive to completion: A large number of students who have historically signed up for MOOCs are observers, 'drop-ins', passive participants and no-shows [Hill, 2013]. The actual number of students who completed to course certification is modest. Further studies reveal that a large majority of students who do complete courses are not there to receive a degree, but to supplement their knowledge. In other words, these are fairly well educated, employed students who are looking for specific training exercises. Potential way forward? Charging a modest sum for courses would tend to drop enrollment substantially, especially from poorer corners of the globe - and limit it to the motivated ones. However, the data overwhelmingly suggests that those who pay the ticket are much more likely to complete the courses they sign up for.

o Lack of a clear business model: MOOCs need to develop a clear sustainable business plan. Most of the students for-profit universities are likely to get are those who cannot enter conventional universities because of poor placement test scores on SAT or GRE. These students view online universities as an easy back-road to success - bypassing the rigor of a conventional four-year college towards employment. There are indeed a lot of jobs that are labor-intensive, so with the right training it is indeed possible for placement in some of these market sectors. These are, however, not high-end jobs, and the return on investment for these students is fairly low. Other than that, the charging of modest sums of money tends to argue against the economy of scale ( $\$ 50$ is a modest sum for a course in the US, but the equivalent Rs. 3000 is still fairly large for an impoverished student in India).

Potential way forward? Perhaps the economics of scale needs to be flexible enough to take advantage of local resources at partner sites and countries. Production costs are considerably cheaper in India, even with its limited resources, due to the abundance of cheap labor, substantial government funding into education, and (unfortunately) the absence of nationwide safety standards such as mandatory training and insurance especially for all students attending on-site venues at partner universities. Some for-profit providers are also dabbling with 'headhunter' fees - charging not just for a certificate of completion, but also for match-making - whereby students pay added charges for successful placement in the industry, and industry pays substantial money to access rosters of highest performing students in key technical courses.

o Lack of clear buy-in from job providers: The headhunting model would really work if there was 
substantial buy-in and sponsorship - not just from philanthropic organizations such as the Gates Foundation, but from high-tech firms such as Google, Intel or Microsoft that employ many skilled students annually. This would, however tie-in directly to a parallel problem, listed next - the lack of standardization and accreditation.

o Lack of standardization and accreditation: A certificate of completion does little to convince job creators that there is actual learning and assimilation happening. It is also incredibly hard to set meaningful questions for thousands of students and grade them properly. Most online course materials tend to provide multiple-choice questions that can in fact be machine graded (GRE exams are administered globally that way). But this means we need to appoint elaborate committees to develop standardized tests for each topic acceptable globally across all universities and countries. Accreditation also becomes hard for questions that require more than intelligent guesses where step-by-step work is needed to be evaluated, and creativity is important. Finally, there is no easy way to proctor these exams - meaning it is not easy to determine who is actually taking an exam, whether they are working in teams, consulting other experts or browsing the internet in parallel to look for answers to questions.

Possible way forward? The job creators have traditionally been left out, but need to be part of this discussion - in fact, part of the sponsorship for courses needs to come from them so that they have a skin in the game. Proctoring could perhaps be done during key exams by asking students to turn on their camera phones while taking exams (still the economy of scale tends to work against it especially in an online environment, unlike GREs where students still go to appointed venues with manual proctoring). Perhaps the way forward is to work with industry and key universities to appoint a committee that sets exams in certain critical topics standardized across the globe. The headhunters then release the information for the highest performing students to the companies, who invite them for an interview. At the interview, the companies administer a second exam - written or oral, to test if the candidate has actually learned the material. Such a multi-tiered process is perhaps expensive to set up initially, but over time could prove beneficial. But the point is that part of the drive must come from the job creators.Journal of Engineering Education Transformations, Special Issue, eISSN 2394-1707 o Branding: This brings us to the very next issue - that of branding. Who sets the exam and who creates the materials? Once there are standardized curricula, say for freshman calculus, developed by highly ranked universities like MIT or Stanford, where is the room for a University of Tennessee or a Texas A\&M to develop calculus based courses? If students get into a MOOC simply by paying and get a certificate in return to get to a company, why wouldn't they automatically flock to an MIT or Stanford? Education then becomes a commodity that comes with packaging and branding and only a few universities could end up monopolizing it.

Possible way forward? Some of this may end up being inevitable. As it stands, different universities are not reinventing calculus. But again, what may make at least some of the universities relevant is the matter of scale - the sheer act of grading and administering the exams (although in the above 'doomsday scenario' for higher education, universities like MIT/Stanford would probably outsource the logistics of exam giving to education companies). However, it is clear that if everything became a MOOC, which may well not happen all the way (but could happen to some degree) as I argue below, each university will need to find ways to invent a clear identity for itself. One way this could play out is as follows. Basic freshman and sophomore courses will probably be outsourced to adjunct faculty or community colleges where teachers are in essence 'graders' - they take standardized curricula (no different from a textbook) and provide additional services such as consulting and tutorials. Senior students and graduates however are offered a buffet of choices that are high-end, emergent topics such as nanotechnology, energy, bioengineering, data science - that are administered by research-active professors who anyway tend to prefer teaching specialized courses that connect research and teaching. It is also conceivable that faculty across multiple universities form a coalition to teach a set of courses connected by a curriculum that is decentralized. For instance, Purdue University launched the NanoHUB-U, which involves courses and lecture notes created by faculty involved in nanoelectronic research across multiple universities. Such specialized courses are still useful and can justify the involvement of faculty active in these areas. o Motivation and 'Bieberification': "We lead students to the fountain of knowledge. Some will drink deeply, some will take a few swallows, and some will just sip. An increasing number will, as at the dentist, merely 
rinse before spitting out". This comment by Dale Arrington may well be the reason MOOCs may not revamp higher education. Ultimately, it is the issue of motivation. Resources outside the classroom have been around for a while, and have been readily accessible since the wide availability of the internet in the mid 1990s. Still, in the last 20 years we have not seen any reduction in college attendees and certainly no reduction of college debt. In many ways, part of Tagore's dream is now here - "...where knowledge is free..". However, it ironically comes at a considerable price. Today's students are bombarded with enormous amounts of data and social media catered to their instant gratification (from Candy Crush to Facebook to music streaming). More data has been generated in the last two or three years than has been generated in the entire history of humanity! (The overabundance of information and its effect on the human psyche is part of the storyline of 'Indiana Jones and the Kingdom of the Crystal Skull' - it is what the futurist Ray Kurzweil calls the 'singularity' event). But what does not come with such an abundance of material is a 'user manual' a clear set of instructions on what data to ignore and what to focus on, how to separate signal from noise, how to navigate one's way amidst the petabytes of information flowing all around. And this is notoriously hard to do, despite the promises of Data Science, because much of knowledge is contextual, influenced by diverse forces from culture - geography and history (space and time).

Where higher education tends to differ from music streaming and Udacity finds it challenging compared to Napster is an essential difference: learning is, ultimately, a participatory activity. Music can be savored at various levels of tangential involvement, while driving or showering or even getting to sleep but active assimilative learning, the kind that isn't rote, is immersive cannot be done in the background. Many students need active engagement driven by human interactions - a teacher strutting around a classroom, making light humor, spewing anecdotes, engaging in Q\&As. A lot of classroom dynamics is ultimately driven by psychology. Many students dislike passive learning - for pretty much the same reason we hate recorded telemessages and frantically look for 'customer service' when we call a business over the telephone.

No doubt some of the courses online can also be made enormously engaging. My own experience as an undergraduate was with UGC courses on Indian TV that were delivered by leading researchers, very oftenin a dull, unrehearsed monotonic style. In contrast, BBC programs on space and science and technology were presented by actors with little authentic knowledge of the subject at hand, but who delivered slick, scripted material that was enormously engaging. Clearly a lot of money went into these courses to ramp up their production value. In the US, we have Bill Nye the Science Guy engaging students with exciting home experiments, Morgan Freeman and Tom Hanks speaking of space exploration in distinctive baritones, Michio Kaku, Carl Sagan and Neil Degrasse Tyson who have become household names, known more for their art at popularizing rather than creating science. Will courses ultimately devolve into popularity contests delivered by celebrities rapped by a Justin Bieber or a Kanye West? Surely we remember the engaging Dino-Rap by Robin Williams teaching archaeology in the movie "Mrs Doubtfire"?

Possible way forward? The first step to realize is that passive education is not for all. Many students need extensive hand-holding, active follow-ups, frequent counseling, motivation and upliftment. They sometimes need therapy. Studies have shown that students in flipped classroom courses tend to do much of their learning late night, in small chunks of 6-9 minutes. Studies also show that $30 \%$ of student time is actually spent in dealing with the coding environment. Courses need to be created taking these factors into account - the relative attention deficiency and hyperactivity of today's students compared to earlier generations, and the ease of initiation into the learning avatar. It is also important to know which activities translate online well. While certain things do scale well, such as training videos and flight simulators what do not scale are those that involve hands-on training - lab-work and field-studies for instance, where mistakes are necessary along with burn-marks and chemical spills, human interactions and social contexts that are necessary to consolidate learning, as well as activities where learning involves the 'Socratic method' of meditating and ruminating - churning information over and over till it settles in optimally.

A related issue is the true value of education in colleges - which isn't just the technical content of courses, but the so-called "soft skills" - especially with term papers and research projects - where one needs to learn how to formulate a problem, cut it down to its essence, how to divide and conquer on the technical side, how to negotiate around road-bumps, how to communicate effectively, and proceed overall from knowledge to wisdom. Many of these soft skills, 
which are essential for an active and diverse job market, do not translate well online.

\section{C. 'Customized Education' as a compromise}

Perhaps a compromise is 'Customized Education' a choice between a simplified bare-bones curriculum primarily with online components and minimal administrative overheads for students looking for skill-based employment, vs face-to-face teaching and hands-on training for those seeking a deeper more engaged and enhanced learning experience, at a higher cost. Lessons not needing human interactions such as flight simulators are already quite popular, as are entire online MBA degrees. The truth is, part of learning is necessarily either rote or involves skill which can to some degree be delivered through repetition online. For many students, this is all they desire - learning useful skills to get a job. Despite the hours of face-to-face lectures that we all offer, there is a nontrivial fraction of students who sit in the back and do not ask questions and are simply not engaged. They are there simply because they feel they have to be there! One can imagine a basic curricular training that delivers knowledge to that group at minimal costs. Beyond that are the curious students - the ones who actually want to engage and learn. For them, an online medium may not suffice. We will still need active human intervention to mould their curiosity into a lifelong quest for learning.

\section{Story of my experiments with online teaching}

Over the years, I experimented with various forms of online teaching. Below are a few lessons learned.

\section{A. Simulation as a tool for trans-disciplinary coursework:}

After the US Congress passed the High Performance Computing and Communication Initiative, simulation is now considered the third branch of science after theory and experiments. Simulation tools carry unique advantages - many courses involve algebra of high complexity that can be distracting for students learning it for the first time. Labs on the other hand are costly and time consuming, and do not scale well in basic engineering courses (except some topics like I-C fab). As a result, many engineering courses become surveys where students are 'told' the design principles. The reasonable alternatives that did not work, plus the joy of discovery, are eliminated.
I introduced elementary engineering topics through numerical simulations, coupled with animations using Cinama3 D. Engineering UGs learn Matlab/MathCAD in their early years, and basic gaming resources like Cinema3D are accessible through about six youtube lectures. In my course "Fundamentals of Nanoelectronics", I armed students with 10-15 line Matlab codes and bypassed weeks of strenuous algebra to arrive at the key concept. My homework's encouraged them to tinker with parameters for better intuition, and deconstruct emerging topics of research through such 'toy' models. I did this for undergraduate courses such as Electromagnetism, Quantum Mechanics and Solid State, through limited algebra (all the gory details were nonetheless available online, but I told them to take a peek only after they've already discovered the results through numerical tinkering). In QM, I equipped students with two codes I wrote- one for the bound states in any 1-D potential well (students can 'discover' eigenstates for a particle-in-a-box, hydrogen atom, harmonic oscillator), while the other solves for resonant states from any 1-D scattering potential (particle on a step, barrier, tunneling, bands and subbands, resonances and transmission). With a little modification, we then expand to real materials such as gated geometries, heterostructures, dipole fields, then proceed to calculate charge, spin and heat current, magnetics and spintronics, thermal flow and thermoelectrics - each taught with no more than 50 lines of Matlab codes over just the first 2-3 weeks, to students with no formal training in quantum mechanics.

The motivation behind these simple 'hands-on' exercises is to provide a path of hierarchical learningthe ability to distinguish between essential, zeroth order information that needs assimilation and retention in one's memory hard drive, vs. 'details' that can be momentarily stored in one's memory cache, and erased unless the student plans to actively pursue that line of study.

B. Hierarchical Learning - Wikipedia meets Hyperphysics:

I believe true understanding happens when the mind can catalogue information into strata - essential vs details. I tried to interpolate between the Hyperphysics site at GSU (http://hyperphysics.phyastr.gsu.edu/hbase/hframe.html) that cascades information along tree diagrams, a d Wikipedia that cascades information into invisible layers. I merged 
the two to create a tree with minimal visible branches upfront (just the large blue, beige and green ovals in Fig. 1), clicking which brought up the deeper branches and ovals. Each small oval is a lesson with a short summary plus an audio lecture recorded offline in Adobe Presenter, with animations in powerpoint synced with voiceover narration and stored as a flash file. Students could pause, rewind, jump between lectures -all the while in context of a tree diagram that shows the logical flow and helps distinguish basic concepts (higher layers) vs details (deeper layers).

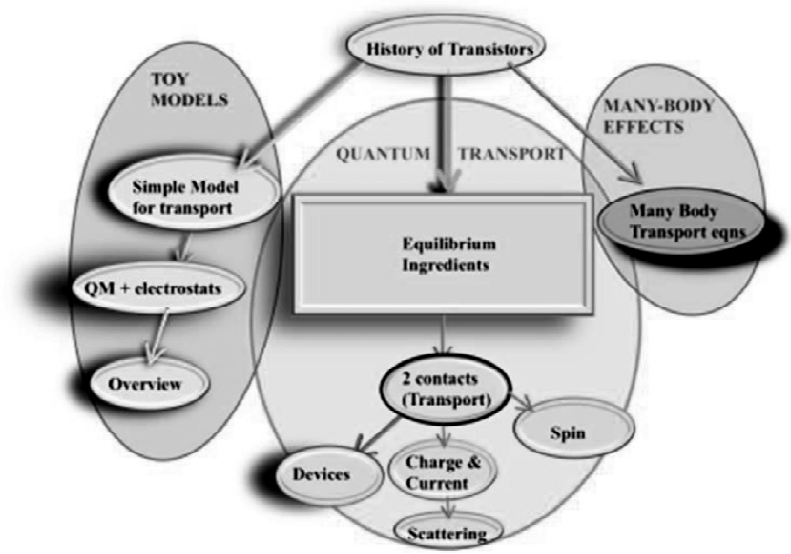

Fig. 1. Course Screenshot showing large up front ovals with clickable that bring up the next layer and so on. At each stage, what comes up is a linked tree that shows the logical flow.

I was expecting student attendance to go down after I made all lectures available online, but found to my pleasant surprise that attendance went up! I believe the explanation is clear. A lot of students DO want to learn, but end up inattentive through a key concept or missing a class and then finding it very difficult to catch up (I faced this myself in college). My lectures provided students a way to catch up quickly with keyed topics and reinsert them into the class. In other words, students controlled their pace of learning and level of detail, to some degree.

\section{C. Asynchronous Blended Teaching:}

This semester, I am teaching a course on asynchronous blended learning. All lectures are provided upfront. They are embedded hierarchically like below. In fact in related tutorials launched on online portals like the NanoHUB, I make my powerpoint lectures self-contained, in that the concepts, their supporting Matlab codes and output data/plots are all embedded (Fig. 2 bottom). Students have access to all lectures and homeworks from day 1 . They have deadlines for turning in each homework and accessing the solution, and instructions on which lectures to cover by which date. We have a weekly quiz to synchronize students periodically (quizzes are delivered online, and are very simple - primarily testing if students are at the right place on the course timeline). I meet students once a week online to give the quiz (our media center helps record those), go over the quiz solution immediately, and summarize key concepts they are supposed to learn that week, which I render in real time with minimal maths. Students ask each other questions on discussion forums - I incentivize participation with performance points and reserve bonus points for excellent, thought-provoking questions. It is incredible how hard some students will work for a few few credit points!

3. The 'art' of teaching. Ultimately, a lot of teaching is experiential and experimental - we try different thing see what works. Part of the experience is perhaps scalable - the part to deal with rote learning or reinforcing. A lot of it - the one that moulds one's curiosity through human interactions, back-andforth dialogue, is probably not easy to scale unless we reach an era of true Artificial Intelligence. This is best emphasized by the following three quotes on education:

'The power or education is seldom of much efficacy except in those happy dispositions where it is almost superfluous'

(Edward Gibbons, 1976 - The decline and fall of the Roman empire Vol $1 \mathrm{Ch} 4$ ).

'The best education consists in immunizing people against systematic attempts at education' (Paul Karl Feyerabend).

'The most important outcome of education is to help students become independent of formal education' (Paul E Gray, President of MIT 19801990).

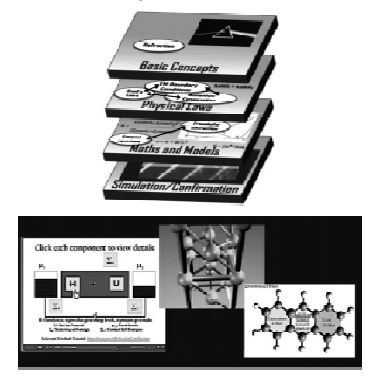

Fig. 2 (Top) Layer diagram showing EM lecture breakdown. (Bottom) Example lecture with clickable links that take one to downloadable files - on the lower right, the left benzene ring has Huckel parameters (.huc), the middle has Matlab codes (.m) and the right has output data (.mat) plottable in Matlab. 


\section{Acknowledgement}

This work was supported by UVa Teaching Resource Center and the US Frontiers of Engineering Education program through the US National Academy of Engineering.

\section{References}

'The strengths and weaknesses of MOOCs: Part 1', T. Bates,(2014)http://www.tonybates.ca/2014/10/19/ the-strengths-and-weaknesses-of-moocs-part-i/

'An early report card on MOOCs', G. A. Fowler (2013), Wall Street Journal Oct 8.

'Some validation of MOOC student patterns graphic', P. Hill (2013) e-Literate, August 30

'HarvardX and MITx: The First Year of Open Online Courses Fall 2012-Summer 2013', A. Hoet al. (2014) (HarvardX and MITx Working Paper No. 1), January 21

'MOOCs: Expectations and Reality',F. Hollands, and D. Tirthali (2014) New York: Columbia University Teachers' College, Center for Benefit-Cost Studies of Education, $211 \mathrm{pp}$
'Whats Wrong With MOOCs, and Why Aren't They Changing the Game in Education?', H. Singh (2014) Wired.com

'MOOCs Are Finally Being Analyzed by Educators ... What's the Verdict?' | EdTech Magazine (www.edtechmagazine.com)

'Arizona State U 'MOOCs for credit' program faces unanswered accreditation questions' | Inside Higher Ed(www.insidehighered.com)

'10 Must-Read Articles about MOOCs', www.talentlms.com

'Moocs: from mania to mundanity' | Times Higher Education (www.timeshighereducation.com)

'Academics Are Down on MOOCs. Business Schools Aren't'- Businessweek (www.bloomberg.com)

'Insights and Trends that Make MOOCs Matter' | EdSurge News (www.edsurge.com)

'8 Myths About MOOCs' | Technology and Learning | Inside Higher Ed (www.insidehighered.com) 